\title{
Sensory features and the reduction of imaginal interference
}

\author{
DOUGLAS L. NELSON, RICHARD C. BORDEN, and JOSEPH W. WHEELER \\ University of South Florida, Tampa, Florida 36620
}

\begin{abstract}
Interference generated among the sensory features of PA stimulus words is not eliminated by processing at the imaginal level. This experiment was planned to test the reverse possibility. Will interference generated by similar imaginal referents be eliminated by sensory overlap between the stimuli and their responses? Thus, stimulus words did or did not evoke similar images, pairs did or did not share initial letters, and lists were acquired either under an instructional set to form interacting images or to repeat the items aloud. The results indicated that the imagery strategy was superior to the repetition strategy in all conditions, and that stimulus interference was statistically eliminated when pairs shared the same initial letters. The findings were interpreted as consistent with a levels of processing conceptualization.
\end{abstract}

Nelson and Brooks (1973), in a series of five experiments, demonstrated that formal similarity among the stimulus words of paired associate (PA) lists impaired acquisition independently of their imagery level. Letter overlap among high imagery, concrete words produced increments in difficulty comparable to those obtained with low imagery, abstract words (Experiments I-III). Interference attributable to similarity was obtained even when the pairs were high imagery words shown at a $3 \mathrm{sec}$ rate and acquired after extensive practice at generating interacting images (Experiments IV-V). These findings were interpreted as indicating that the sensory features of the stimuli, their graphic-phonetic attributes, and their imaginal attributes were both being processed. Thus, even under relatively optimal conditions for coding interacting images, interference generated among the sensory features was not eliminated by processing the pairs as compound images. The present experiment was designed to test the complementary possibility, to determine if the sharing of sensory features among stimuli and their responses would differentially reduce or eliminate interference generated by similar imaginal referents. Thus, stimulus-response pairs did or did not share the same first letter, and stimuli did or did not evoke similar imaginal referents. For example, two of the pairs in one of the overlap-interference lists were JAIL-JET, PRISON-PINE; in the comparable no overlap-interference list, these pairs became JAILMOLE, PRISON-KISS. The stimuli of the no interference lists were chosen to evoke dissimilar images. In addition to these manipulations, instructional set was varied so that half of the subjects in each condition

This research was supported by Grant MH 16360 to the first author from the National Institute for Mental Health and by a University of South Florida Fellowship to the third author. Requests for reprints should be sent to Douglas $L$. Nelson, Department of Psychology, University of South Florida, Tampa Florida 33620 . received interaction imagery instructions, and half received overt repetition instructions. Acquisition strategy was manipulated in an attempt to bias coding, respectively, towards processing either the imaginal or the sensory referents of the items.

When stimulus-response pairs did not share initial letters, it seemed reasonable tc expect that the imagery set would produce faster acquisition relative to the repetition set (Paivio \& Yuille, 1969), and that stimulus interference would be present under both instructional sets. The latter expectation was predicted on the assumption that subjects utilizing the repetition instructions would not continue to process the pairs at only the sensory level. But, rather, that they would begin coding semantic or imaginal information inherent in the stimuli, and that the processing of these features would produce interference (Paivio \& Yuille, 1969). When stimuli did share first letters with their responses, three theoretically interesting outcomes seemed plausible. All of these outcomes were based on the assumption that overlapping first letters would facilitate performance, which would be consistent with previous findings (Nelson, Fosselman, \& Peebles, 1971). This facilitation, therefore, was expected to be mostly a matter of degree, potentially varying both as a function of instructional set and presence of stimulus interference. First, overlap among initial letters might eliminate both stimulus interference and instructional effects. This outcome could mean that the pairs were being processed at only the prior sensory level under each instructional set, and that further stimulus elaboration is not carried out because of the apparent effectiveness of this coding. Second, sensory overlap might eliminate interference when subjects are operating under the repetition set, but not when they are coding interacting images. If performance remained superior with the imagery strategy, this finding would imply that imaginal referents are being processed and that, once an 
Table 1

Mean Errors (Estimates of Standard Deviations) as a Function of Stimulus-Response Overlap, Stimulus Interference, and Type of Instructions

\begin{tabular}{|c|c|c|c|c|c|c|c|c|}
\hline \multirow{4}{*}{$\frac{\text { Instructions }}{\text { Imagery }}$} & \multicolumn{8}{|c|}{ Stimulus-Response Overlap } \\
\hline & \multicolumn{4}{|c|}{ First Letter Overlap } & \multicolumn{4}{|c|}{ No Overlap } \\
\hline & \multicolumn{2}{|c|}{ Interference } & \multicolumn{2}{|c|}{ No Interference } & \multicolumn{2}{|c|}{ Interference } & \multicolumn{2}{|c|}{ No Interference } \\
\hline & 9.31 & $(5.17)$ & 6.19 & ( 4.07) & 22.00 & $(10.98)$ & 10.00 & $(7.09)$ \\
\hline Repetition & 18.25 & $(10.32)$ & 15.06 & (13.43) & 39.12 & $(21.63)$ & 25.31 & (16.33) \\
\hline M & 13.78 & & 10.62 & & 30.56 & & 17.66 & \\
\hline
\end{tabular}

image is activated, sensory information inherent in the physical stimulus is no longer an effective mnemonic. Thus, when the stimulus has been coded at the meaning level, sensory information may not be accessible (e.g., Craik \& Lockhart, 1972). Third, the stimulus interference effect might be eliminated under both instructional sets but, nevertheless, performance under the imagery set may remain superior. This finding would suggest that both sensory and image referents were being processed under the interaction imagery instructional set. Furthermore, this outcome would raise the interesting issue of why, in the Nelson and Brooks (1973) experiment, processing differentiating imaginal referents did not eliminate interference generated at the sensory level whereas processing differentiating sensory attributes eliminated interference generated at the imaginal level.

\section{METHOD}

\section{Design and Lists}

The experimental design was a $2^{4}$ between-subjects factorial. Presence-absence of first letter stimulus-response overlap was combined with presence-absence of stimulus interference, type of instructions, and male experimenters.

In the overlap conditions, each stimulus-response pair shared only the same first letter, e.g., JAIL- JET, FOAM-FLAG. To form the PA pairs for the no overlap conditions, the responses of the overlap lists were reassigned to other stimuli in the list, e.g., JAIL-MOLE, FOAM-JET. All responses were drawn from the Colorado concreteness and imagery norms, and all were high imagery words having an average rating of 5.99 . The PA stimuli for the interference lists consisted of seven sets of synonyms selected so that, in the judgment of colleagues, an image generated for one member of the set was highly similar to that produced for the other member of that set. Two different, but apparently comparable lists were constructed for each of these conditions. For List 1 , the 14 stimuli were JAIL, PRISON; FOAM, LATHER; DWARF, MIDGET; BARREL, KEG; GROVE, ORCHARD; HARE, RABBIT; AUTO, CAR; and, for List 2, these items were BONNET, HAT; CART, WAGON; DIRT, SOIL; FOG, MIST; GEM, JEWEL; LAKE, POND; ROBBER, THIEF. The stimuli for the two no interference lists were selected by taking, from each list, one member of the similar set from each of the interference lists, e.g., JAIL, BONNET, FOAM, CART, etc. In fact, the no interference lists were constructed by taking one-half of the pairs from each interfering list. This procedure represented a partial control for differences in word pair characteristics for the two interference conditions.

In all lists, obvious semantic or associative relationships between stimuli and their responses were eliminated and, in addition, formal similarity among the stimuli and among the responses was minimized.

\section{Procedure}

Each subject acquired a single list by the study-test method in an individual session. All items were presented via a Kodak Carousel slide projector driven by an auxiliary timer at a $3: 3 \mathrm{sec}$ rate. The intervals between study and test phases and between trials were also $3 \mathrm{sec}$. Practice was continued for 25 trials or four consecutive trials without an error, whichever came first. Sequences of pairs and of test stimuli were presented in four separate and unsystematically varying orders that were changed for each subject in each condition. The only restriction was that at least four tests had to intervene between the presentation of a pair and its test.

The interaction imagery instructions indicated that the task was to learn what words went together, and that the most efficient way to accomplish this goal was to mentally construct a picture containing images of both of the words acting together in some way. The experimenter provided several examples of this concept, and then asked the subject to generate interacting images for several other pairs. Appropriate feedback was provided to insure understanding. The repetition instructions indicated that the most efficient way to learn the pairs was to repreat them aloud as often as time allowed. Examples were provided by the experimenter. All subjects were required to pronounce each test word aloud before attempting response anticipation. Following the instructions, all subjects learned the same three pair proper name-verb practice list before undertaing the the experimental task.

\section{Subjects}

There was a total of 128 subjects, drawn from courses in introductory psychology, with 16 assigned to each of the principal conditions, 8 to each list. For each experimenter, these subjects were assigned to treatments in blocks of 16 , with one subject from each treatment per block. Assignment within blocks was determined by a table of random numbers.

\section{RESULTS}

Table 1 presents means of errors, including omission and comission, obtained in achieving the acquisition criterion for the principal conditions of the experiment. An analysis of variance of these data indicated that main effects of stimulus-response overlap, stimulus interference, and type of instructions were all significant at $p<.01$ with Fs $(1 / 120)$ of, respectively, 29.37, 13.36 , and 32.70 . Thus, in every condition, performance was facilitated if stimuli shared first letters with their responses and if the instructions articulated the interaction imagery as compared to the repetition strategy. Performance was impaired if sets of stimuli 
were synonyms. However, as shown in the bottom row of Table 1, the degree of impairment was dependent upon the presence of sharing among the pairs. The Overlap by Interference interaction was significant, $F(1 / 120=4.92, p<.05$, and Fisher's least significant difference for this source was 6.15 . Therefore, the stimulus interference effect was reliably obtained only when there was no sensory overlap among pairs, not when stimuli shared first letters with their responses. Of the remaining sources, only the Overlap by Instructions interaction achieved an $F$ of greater than unity. Although there was a tendency for sharing to produce greater facilitation in the repetition condition, this interaction failed to achieve the criterion of statistical significance, $F(1 / 120)=2.77$. Because of the differences in variance estimates associated with manipulations of instructions, separate analyses of variance were performed for each instructional set. The statistical results simply confirmed those of the overall analysis. Furthermore, examination of trial by trial performance (94\% of the subjects reached criterion by Trial 10) indicated that the patterns of results obtained with pooled errors was maintained throughout acquisition.

An analysis of stimulus confusion errors obtained in the interference conditions corroborated these findings. A stimulus confusion occurred when a response belonging to one stimulus was given to its synonymous pairmate. For the overlap conditions, mean stimulus confusions were .38 and.44, respectively, for imagery and for repetition instructions; for the no overlap conditions, these respective means were 3.75 and 3.06 . An analysis of variance of these conditions indicated that significantly fewer confusions were obtained when the pairs overlapped in first letter, $F(1 / 60=16.30$, $\mathrm{p}<.01$. Neither type of instructions nor the interaction between this variable and overlap was significant, both Fs $<1$. Thus, when MOLE was paired with JAIL, the subjects were much more likely to give it as a response to PRISON than when JET was paired with JAIL.

\section{DISCUSSION}

The subjects coding under the influence of the interaction imagery instructions apparently were not coding at only the imaginal level, nor did they appear to be processing the pairs at only the sensory level. When the stimulus-response pairs shared the same first letter, imagery instructions were superior to repetition instructions and, of equal importance, the interference effect generated by sets of stimulus synonyms was statistically eliminated under each instructional set. However, even when pairs overlapped in initial letters, more errors were obtained in the interference than in the no interference conditions. This numerical difference suggests that it would be more accurate to characterize these results as representing an attenuation of interference rather than as an elimination of it. Thus, in considering the entire pattern of findings, this experiment indicates that both sensory and imaginal referents of the items were being processed when the subjects were operating under the guidance of the instructional set to form compound images. Even though imaginal codes were being processed, information inherent in the sensory code served to facilitate performance and to attenuate interference generated at the imaginal or meaning level. Sensory information apparently did not simply become inaccessible as more elaborative codes were developed. This result is consistent with previous findings and some theoretical interpretations of the effectiveness of earlier levels of processing (e.g., Nelson, Wheeler, Borden, \& Brooks, 1974; Posner \& Warren, 1972), but not with others (e.g., Craik \& Lockhart, 1972).

The sharing of sensory attributes may have reduced interference produced at higher levels because the overlapping initial letters enhanced the development of interacting images. Intuitively, this possibility does not seem likely. Why would it be easier to generate a compound image for JAIL-JET than for JAIL-MOLE? Alternatively, and consistent with the general concept of levels of processing, it seems reasonable to presume that prior to developing an interacting image, the nominal stimulus and the nominal response must first be identified. This identification process requires the coding of graphic and phonetic features of each of the items so that their respective name codes can be activated or primed. Given that the priming of sensory features produces a relatively durable code (Nelson \& Rothbart, 1972), this information could serve as a means for relating stimuli and their responses, just as interacting images serve as mnemonics for relating stimuli and their responses. In the no interference conditions of this experiment, either sharing of first letters or an instructional set to form interacting images served to facilitate retrieval. Of all of the conditions, fewest errors were obtained when both of these contingencies were in effect, when pairs overlapping in initial letters were coded under the imagery set. In the interference conditions, first letter cues inherent in the stimuli served to facilitate retrieval in general and to attenuate meaning interference in particular. Moreover, given the priming of sensory attributes during coding, these cues might serve to reduce interference at the point of decoding. For example, suppose that when JAIL has been shown at test, the response for PRISON is recalled, PINE. A stimulus confusion has occurred. However, in noting that the recalled word PINE does not begin with the letter "J," the retrieval process may be reiterated, thereby decreasing the likelihood that the correct response, JET, will be recalled, and thereby decreasing the likelihood that a stimulus confusion will occur. This mechanism also might explain why sensory interference was not differentially attenuated by processing imaginal (Nelson \& Brooks, 1973) or semantic-associative 
referents (Nelson \& Borden, 1973). In those experiments, if the processing of the sensory features of the stimulus results in contact with a formally similar functional referent, that is, if a stimulus confusion occurs, an error is relatively more likely, since the decoded response does not share specific differentiating cues with that stimulus. By contrast, in the present experiment, the sensory information inherent in the decoded response could be processed to determine if it matched that contained in the stimulus.

\section{REFERENCES}

Craik, F. M., \& Lockhart, R. S. Levels of processing: A tramew ork for memory research. Journal of Verbal Learning and Verbal Behavior, 1972, 11,671-684.

Nelson, D. L., \& Borden, R. C. Effect of meaning on processing of phonetic features of words. Journal of Experimental Psych ology, 1973, 101, 373-375.

Nelson, D. L., \& Brooks, D. H. Independence of phonetic and imaginal features. Journal of Experimental Psychology, 1973, 97, 1-7.

Nelson, D. L., Fosselman, J. R., \& Peebles, J. Words as sets of features: Processing phonological cues. Journal of Experimental Psychology, 1971, 87, 361-366.

Nelson. D. L.. Wheeler. J. W., Borden, R. C.. \& Brooks, D. H. Levels of processing and cueing: Sensory vs, meaning features. Journal of Experimental Psychology, 1974, 103.971-977.

Nelson, T. O., \& Rothbart, R. Acoustic savings tor items forgotten from memory. Journal of Experimental Psychology, $1972,93,357360$.

Paivio, A., \& Yuille, J. C. Changes in associative strategies in paired-associate learning over trials as a function of word imagery and type of set. Journal of Experimental Psychology, $1969,79,458-463$.

Posner, M. I., \& Warren, R. E. Traces, concepts, and conscious constructions. In A. W. Melton \& E. Martin (Eds.), Coding processes in human memory. Washington, D.C.: Winston, 1972.

(Received for publication June 7,1974; revision received, July $31,1974$. 